\title{
Desnutrición y rendimiento académico de los alumnos del primer al sexto grado de primaria de la I. E. E. 18003 "Santa Rosa". Chachapoyas-2017
}

\section{Malnutrition and academic performance of students from the first to sixth grade of primary school of the I. E. E. 18003 "Santa Rosa". Chachapoyas-2017}

\author{
Luis Felipe Gonzales Llontop ${ }^{1}$
}

\begin{abstract}
RESUMEN
El estudio fue encaminado a evaluar la desnutrición y rendimiento académico de los alumnos del primer al sexto grado de primaria de la I. E. E. 18003 "Santa Rosa". Chachapoyas-2017. Fue un análisis de tipo correlacional con enfoque cuantitativo, en donde nos propusimos el objetivo de conocer la relación entre la desnutrición y rendimiento académico de los escolares de la institución educativa "Santa Rosa", ubicada en el distrito de Chachapoyas. La muestra estuvo constituida por 66 niños del primer al sexto grado de primaria del año 2017. Se tomaron los datos mediante una ficha de recolección tanto para determinar el estado nutricional y el rendimiento académico; se procedió a pesar y a tallar según el IMC y clasificar la desnutrición de los escolares. Se tomó los calificativos de cada estudiante referente al año académico 2017. Los resultados muestran al relacionar el estado nutricional con el rendimiento académico que el 42,44\% mostró bajo peso con rendimiento académico alto; los escolares presentaron peso normal $(37,88 \%)$ y con sobre peso $(10,61 \%)$ un alto rendimiento académico mientras que el $1,52 \%$ con sobre peso mostró bajo rendimiento académico.Se concluye que no existe una relación significativa entre ambas variables estudiadas.
\end{abstract}

Palabras clave: desnutrición, rendimiento académico, estudiantes

\begin{abstract}
The study was aimed at assessing malnutrition and academic performance of students from the first to sixth grade of primary school of the I. E. 18003 "Santa Rosa". Chachapoyas-2017. It was a correlational analysis with a quantitative approach, where we set ourselves the objective of knowing the relationship between malnutrition and academic performance of schoolchildren of the educational institution "Santa Rosa", located in the Chachapoyas district. The sample was constituted by 66 children from the first to the sixth grade of the year 2017. The data were taken through a collection card to determine the nutritional status and academic performance; We proceeded to weigh and carve according to the BMI and classify malnutrition of schoolchildren. The qualifications of each student were taken referring to the academic year 2017. The results show when relating the nutritional status with the academic performance that $42.44 \%$ showed low weight with high academic performance; the students presented normal weight $(37.88 \%)$ and with overweight $(10.61 \%)$ a high academic performance while the $1.52 \%$ with overweight showed low academic performance. It is concluded that there is no significant relationship between both variables studied.
\end{abstract}

Keywords: malnutrition, academic performance, students.

'Doctor Biólogo. Docente asociado de la Facultad de Ingeniería de Sistemas y Mecánica Eléctrica de la Universidad Nacional Toribio Rodríguez de Mendoza de Amazonas. Doctor en Ciencias biomédicas. Correo electrónico: luifego@hotmail.com 


\section{INTRODUCCIÓN}

En pleno proceso de globalización, el Perú continúa siendo un país en vías de desarrollo y aún se mantiene a nivel sudamericano y a nivel mundial como uno de los países de pobreza extrema. A ello hay que añadirle el grave proceso de desnutrición que algunas áreas geográficas de su territorio vienen padeciendo en especial la niñez.

Las políticas de los gobiernos de turno no han respondido a la resolución de este dilema desde hace muchas décadas. El factor socioeconómico juega un rol importante y decisivo en la alimentación adecuada y digna en cada peruano, en cada familia y en toda la sociedad. Si esta no es de prioridad para los intereses de los gobernantes será demasiado tarde su reacción para liberarse del estancamiento, de la miseria, la desigualdad y la injustica. Pronto se verá sus frutos como el bajo rendimiento académico e intelectual de sus jóvenes y niños y la dependencia total de una nación de otras culturas de la cual será difícil desprenderse.

La desnutrición en el Perú y a nivel mundial establece un gran dilema de bienestar orgánico para todo ser humano especialmente los niños, y ello se debe a muchos factores causales como: enfermedades infecciosas y una alimentación inadecuada, estas causas inmediatas, a su vez, son consecuencias de factores biológicos, insuficiente información y educación de los padres, cuidado inadecuado de la madre al niño, acceso limitado a los servicios de salud y deficiente saneamiento básico (Largo, 2006).

Amazonas es uno de los departamentos donde los diferentes índices de desnutrición infantil se ubican muy por encima del nivel nacional. La falta de peso para la talla o desnutrición aguda, afecta al $8 \%$ de los niños de Amazonas, siendo este un indicador de desnutrición reciente por efecto del deterioro de la alimentación y/o de presencia de enfermedades (Guyton, 2014). La desnutrición aguda de Amazonas tiene un mayor nivel entre los niños residentes el área rural y entre los niños de 36 a 47 meses de edad (MINSA, 2011).

Durante las últimas décadas, a nivel, internacional; el binomio madre-estudiante, no es un tema nuevo en diferentes países del mundo, tal es el caso de Uruguay que en el año 2006, la cantidad de madres universitarias alcanzó un porcentaje total de 30,4\% (Varela, 2006). En cambio en España el porcentaje encontrado fue de $19,2 \%$, pero en el año 2004 este porcentaje se incrementó hasta el 22,1\% (San Segundo, 2006).
Por esta justa razón; se dice que muchos de nuestros niños de 3 a 5 años: tienen desnutrición aguda, debido a que los principales factores que ocasionan este problema de salud infantil se encuentran el factor socioeconómico como uno de los principales obstáculos que impiden el progreso del país, De esto se desprende efectos como problemas de aprendizaje en la escuela, bajo rendimiento escolar y mala captación de los conocimientos impartidos por los docentes; ya que son pocas las veces que los niños de nuestro país alcanzan un buen nivel de coeficiente intelectual. Entonces nuestra realidad es que poseemos un bajo nivel intelectual a nivel mundial (Mundaca, 2011).

En el Perú se ha observado que el rendimiento académico en promedio es de medio a bajo, los factores que delimitan el proceso enseñanza aprendizaje no se manejan adecuadamente es por eso que se ve reflejado en bajo nivel de rendimiento académico y está presente en todos los lugares del Perú especialmente Amazonas considerada como una región con extremada pobreza y hasta hoy día no se conoce con exactitud y precisión cuales son los factores predominantes que determinan el rendimiento académico de los estudiantes. El rendimiento académico está incrementándose en algunas universidades nacionales como la Universidad Nacional del Callao, pero que en su mayoría el bajo nivel de rendimiento académico aún se mantiene (Toro, V. 2011).

En tal sentido en el cual existe un entorno sumamente complejo acuden personas de todo tipo de condición social, económica, de diferentes localidades, personalidades, culturas, estigmas, creencias, costumbres, con sentimientos, ideas, modos de actuar diferentes; esto ha traído como consecuencia que durante muchos años no se ha tomado en cuenta estos factores decisivos que si intervienen en el rendimiento académico.

Nos propusimos en este estudio determinar la desnutrición y su relación con el rendimiento académico de los alumnos del primer al sexto grado de primaria de la I. E. E. 18003 Santa Rosa. Chachapoyas-2017.

\section{MATERIAL Y MÉTODOS}

El estudio fue disciplinario y cuantitativo de tipo no experimental, transversal descriptivo correlacional; es no experimental porque no se manipularan las variables de estudio. Es transversal; porque las variables de estudio se darán en un momento dado (Sampieri etal., 2015). 


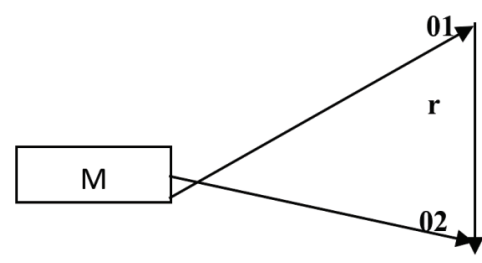

Dónde:

M: Muestra

$\mathrm{O}_{1}=$ Desnutrición

$\mathrm{O}_{2}=$ Rendimiento académico

$r=$ Representa la relación entre las variables.

\section{POBLACIÓN MUESTRA Y MUESTREO}

Población: Con los mismos datos estadísticos proporcionados por la IEE 18003 Santa Rosa se tuvo una población asignada de niños de 5 a 10 años que fue de 66 niños.

Muestra: La muestra estuvo constituida por 66 niños.

Muestreo: Se trabajó con una cantidad entera.

\section{METODOS TÉCNICAS E INSTRUMENTOS DE RECOLECCIÓN DE DATOS}

\section{-Variable 1: Desnutrición}

La medición de la variable se realizó de la siguiente manera:

Se usó la técnica de antropometría, el método de la Tabla del IMC en niño y se usó el instrumento de clasificación de Waterloo.

-Variable 2: Rendimiento académico

Método: se usó el método de la observación (Canales, 2006; Beltrán, 2015)

Técnica: se usó los registros de evaluación (Canales, 2006).

Instrumentos: Se utilizó la ficha de recolección de datos elaborada por el autor sobre rendimiento académico.

\section{ANÁLISIS DE DATOS}

En la valoración estadística se tomó en cuenta los datos que fueron procesados mediante la prueba $\mathrm{Chi}$ cuadrado para evaluar la relación entre variables de estudio, utilizándose el programa estadístico SSPS versión 21,0 y la hoja de cálculo Excel 2013.

\section{RESULTADOS}

Tabla 1: Grado de desnutrición y rendimiento académico de los alumnos del primer al sexto grado de primaria de la I.E.E. 18003 "Santa Rosa" de Chachapoyas -2017 .

\begin{tabular}{|c|c|c|c|c|c|c|c|c|c|}
\hline \multirow{2}{*}{\multicolumn{2}{|c|}{$\begin{array}{l}\text { Rendimiento } \\
\text { Académico }\end{array}$}} & \multicolumn{7}{|c|}{ DIAGNÓSTICO NUTRICIONAL } & \multirow[t]{2}{*}{ Total } \\
\hline & & AGUDA $L^{\circ}$ & $\mathrm{DC} \mathrm{C}^{\circ}$ & DCRE & DCRID & NORMAL & OBESO $\mathrm{L}^{\circ}$ & $S \mathbb{P}$ & \\
\hline \multirow[t]{2}{*}{ A } & $n^{\circ}$ & 3 & 9 & 13 & 3 & 25 & 2 & 7 & 62 \\
\hline & $\%$ & 4,55 & 13,64 & 19,70 & 4,55 & 37,88 & 3,03 & 10,61 & 93,94 \\
\hline \multirow[t]{2}{*}{ B } & $n^{\circ}$ & 0 & 0 & 1 & 0 & 0 & 0 & 0 & 1 \\
\hline & $\%$ & 0,0 & 0,0 & 1,52 & 0,0 & 0,0 & 0,0 & 0,0 & 1,52 \\
\hline \multirow[t]{2}{*}{$\mathrm{C}$} & $n^{\circ}$ & 0 & 1 & 0 & 1 & 0 & 0 & 1 & 3 \\
\hline & $\%$ & 0,0 & 1,52 & 0,0 & 1,52 & 0,0 & 0,0 & 1,52 & 4,55 \\
\hline Total & & 3 & 10 & 14 & 4 & 25 & 2 & 8 & 66 \\
\hline
\end{tabular}

Fuente: Elaboración propia.

$X_{c}^{2}=11.488 \quad$ g. $1 .=12 \mathrm{p}=0.488>0.05$

Se acepta Ho, concluyéndose con el $95 \%$ de confianza que el rendimiento académico de los alumnos es independiente del grado de desnutrición.

Tabla 2: Grado de desnutrición de los alumnos del primer al sexto grado de primaria de la I.E.E. 18003 "Santa Rosa" de Chachapoyas-2017.

\begin{tabular}{|c|c|c|c|c|c|c|c|c|c|c|}
\hline $\begin{array}{c}\text { GRADO DE } \\
\text { ESTUDIOS }\end{array}$ & & \multicolumn{7}{|c|}{ DIAGNÓSTICO NUTRICIONAL } & \multicolumn{1}{c|}{ Total } \\
\hline PRIMERO & $n^{\circ}$ & 1 & 1 & 1 & 0 & 5 & 0 & 0 & 8 \\
\hline & $\%$ & 12.5 & 12.5 & 12.5 & 0.0 & 62.5 & 0.0 & 0.0 & 100.0 \\
\hline SEGUNDO & $n^{\circ}$ & 0 & 1 & 4 & 0 & 3 & 0 & 1 & 9 \\
\hline & $\%$ & 0.0 & 11.1 & 44.4 & 0.0 & 33.3 & 0.0 & 11.1 & 100.0 \\
\hline TERCERO & $n^{\circ}$ & 1 & 2 & 2 & 2 & 8 & 1 & 2 & 18 \\
\hline & $\%$ & 5.6 & 11.1 & 11.1 & 11.1 & 44.4 & 5.6 & 11.1 & 100.0 \\
\hline CUARTO & $n^{\circ}$ & 0 & 2 & 4 & 1 & 3 & 0 & 3 & 13 \\
\hline & $\%$ & 0.0 & 15.4 & 30.8 & 7.7 & 23.1 & 0.0 & 23.1 & 100.0 \\
\hline QUINTO & $n^{\circ}$ & 0 & 1 & 0 & 1 & 2 & 0 & 1 & 5 \\
\hline & $\%$ & 0.0 & 20.0 & 0.0 & 20.0 & 40.0 & 0.0 & 20.0 & 100.0 \\
\hline SEXTO & $n^{\circ}$ & 1 & 3 & 3 & 0 & 4 & 1 & 1 & 13 \\
\hline Total & & 7.7 & 23.1 & 23.1 & 0.0 & 30.8 & 7.7 & 7.7 & 100.0 \\
\hline
\end{tabular}

Fuente: Elaboración propia

De los alumnos del primer grado de primaria, el $12,5 \%$ tiene desnutrición aguda; el $12,5 \%$ tiene $\mathrm{DCI}^{\circ}$; el $12,5 \%$ tiene DCRI $^{\circ}$; el $0 \%$ tiene DCRII ${ }^{\circ}$; el 62,5\% normal; el $0 \%$ obeso y el $0 \% \mathrm{~S} / \mathrm{P}$.

De los alumnos del segundo grado de primaria, el 0\% tiene desnutrición aguda; el $11,1 \%$ tiene $\mathrm{DCI}^{\circ}$; el $44,4 \%$ tiene DCRI $^{\circ}$; el $0 \%$ tiene DCRII $^{\circ}$; el 33,3\% normal; el $0 \%$ obeso y el $11,1 \% \mathrm{~S} / \mathrm{P}$.

De los alumnos del tercer grado de primaria, el 5,6\% tiene desnutrición aguda; el 11,1\% tiene $\mathrm{DCI}^{\circ}$; el $11,1 \%$ tiene DCRI $^{\circ}$; el $11,1 \%$ tiene DCRII ${ }^{\circ}$; el 44,4\% normal; el 5,6\% obeso y el $11,1 \% \mathrm{~S} / \mathrm{P}$.

-De los alumnos del cuarto grado de primaria, el $0 \%$ tiene desnutrición aguda; el 15,4\% tiene $\mathrm{DCI}^{\circ}$; el $30,8 \%$ tiene DCRI $^{\circ}$; el 7,7\% tiene DCRII ${ }^{\circ}$; el 23,1\% normal; el $0 \%$ obeso y el $23,1 \% \mathrm{~S} / \mathrm{P}$.

De los alumnos del quinto grado de primaria, el $0 \%$ 
tiene desnutrición aguda; el 20,0\% tiene $\mathrm{DCI}^{\circ}$; el $0 \%$ tiene DCRI ${ }^{\circ}$; el 20\% tiene DCRII ${ }^{\circ}$; el 40,0\% normal; el $0 \%$ obeso y el $20,0 \% \mathrm{~S} / \mathrm{P}$.

-De los alumnos del sexto grado de primaria, el 7,7\% tiene desnutrición aguda; el 23,1\% tiene $\mathrm{DCI}^{\circ}$; el $23,1 \%$ tiene $\mathrm{DCRI}^{\circ}$; el $0 \%$ tiene $\mathrm{DCRII}^{\circ}$; el $30,8 \%$ normal; el 7,7\% obeso y el 7,7\% S/P.

Tabla 3: Rendimiento Académico de los alumnos del primero al sexto grado de primaria de la I.E.E. 18003 "Santa Rosa" de Chachapoyas - 2017.

\begin{tabular}{|c|c|c|c|c|c|c|c|c|}
\hline \multirow[t]{3}{*}{ GRADO DEESTUDIOS } & & & \multicolumn{3}{|c|}{ RENDIMIENTO ACADÉMICO } & & \multirow{3}{*}{$\begin{array}{c}\text { Total } \\
n^{0} \\
\end{array}$} & \multirow[b]{3}{*}{$\%$} \\
\hline & \multicolumn{2}{|c|}{ A } & \multicolumn{2}{|c|}{$C$} & \multirow{2}{*}{$\begin{array}{l}D \\
n^{0}\end{array}$} & & & \\
\hline & $n^{0}$ & $\%$ & $n^{0}$ & $\%$ & & $\%$ & & \\
\hline PRIMERO & 7 & 11.3 & 1 & 100.0 & 0 & 0.0 & 8 & 12.1 \\
\hline SEGUNDO & 9 & 14.5 & 0 & 0.0 & 0 & 0.0 & 9 & 13.6 \\
\hline TERCERO & 15 & 24.2 & 0 & 0.0 & 3 & 100.0 & 18 & 27.3 \\
\hline CUARTO & 13 & 21.0 & 0 & 0.0 & 0 & 0.0 & 13 & 19.7 \\
\hline QUINTO & 5 & 8.1 & 0 & 0.0 & 0 & 0.0 & 5 & 7.6 \\
\hline SEXTO & 13 & 21.0 & 0 & 0.0 & 0 & 0.0 & 13 & 19.7 \\
\hline Total & 62 & 100.0 & 1 & 100.0 & 3 & 100.0 & 66 & 100.0 \\
\hline
\end{tabular}

Fuente: Elaboración propia.

De los alumnos que obtuvieron $\mathrm{A}$ en su rendimiento académico, el 11.3\% son del primer grado; el 14.5\% segundo; el $24.2 \%$ tercero; el $21.0 \%$ cuarto; el $8.1 \%$ quinto; y el $21.0 \%$ sexto grado de primaria.

De los alumnos que obtuvieron $\mathrm{C}$ en su rendimiento académico, el $100.0 \%$ son del primer grado de primaria.

De los alumnos que obtuvieron $\mathrm{D}$ en su rendimiento académico, el $100.0 \%$ son del tercer grado de primaria.

\section{DISCUSIÓN}

En la tabla 1 y en la figura 1 se evaluó el grado de desnutrición y rendimiento académico de los alumnos presentando las siguientes características: el $42,44 \%$ mostró bajo peso con rendimiento académico alto; los escolares presentaron peso normal $(37,88 \%)$ y con sobre peso $(10,61 \%)$ un alto rendimiento académico mientras que el $1,52 \%$ con sobre peso mostró bajo rendimiento académico. Hay que destacar que de las seis aulas de los alumnos el $3,04 \%$ y el $1,52 \%$ con bajo peso exhibieron bajo rendimiento académico $(4,55 \%)$ y rendimiento académico regular $(1,52 \%)$ respectivamente.
Nuestros resultados coinciden con un estudio titulado "Relación entre el estado nutricional y el rendimiento académico en estudiantes de la institución educativa José Enrique Celis Bardales. Tarapoto, Mayo Diciembre 2011", donde se observó que de un 100\%, el $42.9 \%$ presentan un estado nutricional con bajo peso, y a su vez presentaron un rendimiento académico regular en casi todas sus asignaturas. Mientras que el $39.7 \%$ presentó un estado nutricional normal, con un rendimiento académico también regular, y el $15.9 \%$ de los escolares que presentaron sobrepeso presentaron también un rendimiento académico regular, y solo el $1.5 \%$ de los escolares que presentaron obesidad también presentaron un rendimiento académico regular. Se concluye que no existe una relación significativa entre ambas variables estudiadas.

Analizamos la tabla 2 y en la figura 2 referente al estado nutricional de los alumnos estos exhibieron al $100 \%$ : bajo peso (47\%), peso normal $(37,88 \%)$, obeso $(3,03 \%)$ y con sobre peso $(12,09 \%)$. Resultados muy semejantes se hallaron en el estudio realizado en el año 2011 en la Institución Educativa "José Enrique Celis Bardales" de Tarapoto donde se presentó el estado nutricional de los escolares siendo el mayor porcentaje con un estado nutricional de bajo peso en una sola aula con un $18.2 \%$ mientras el estado nutricional de los escolares tanto del 5 to y 6to grado (dos aulas), en donde se observa que el $39.7 \%$ presenta un estado nutricional normal mientras que el $42.9 \%$ presentan un Bajo Peso.

Respecto al rendimiento académico (tabla 3 y figura 3) podemos señalar que los resultados muestran que los estudiantes de la I. E. "Santa Rosa" presentaron: un alto rendimiento $(93,94 \%)$, un regular rendimiento $(1,52 \%)$ y un bajo rendimiento $(4,55 \%)$ a pesar de gozar de un alto grado de desnutrición en diferentes formas. Cotejando el nivel de rendimiento académico encontrado en los estudiantes de la Institución Educativa "José Enrique Celis Bardales" de Tarapoto, se observó que la mayoría de sus escolares presentan un rendimiento académico regular, a excepción de la asignatura de Matemática donde 2 secciones (5to A y 5to C) presentan un rendimiento académico Alto, mientras que el resto de escolares presenta un rendimiento académico regular casi en todas sus asignaturas. El sustento teórico refiere que el rendimiento escolar es la capacidad del estudiante de rendir eficazmente en las actividades académicas de acuerdo a la edad, la cual se ve afectada cuando existen factores que desfavorecen este rendimiento evitando que rindan al $100 \%$.

Los agentes económicos y sociales que influyen en la 
escolaridad han sido objeto de numerosos ensayos; sin embargo, existe reducida información sobre los efectos que tienen la nutrición y la salud sobre el rendimiento escolar. Es posible que esta escasez de información haya contribuido a que, en sus políticas educativas, la mayoría de las naciones hayan prestado poca atención a la etapa nutricional y de salud de los alumnos (Pollit, 2005).

En las tablas del 4 al 9, al correlacionarse las dos variables mediante el Chi cuadrado para descubrir el nivel de significancia, se concluye que no existe relación estadísticamente significativa entre ambas variables de estudios por lo que se rechaza la hipótesis alterna de investigación.

\section{CONCLUSIONES}

Los estudiantes de la I. E. "Santa Rosa” presentaron: un alto rendimiento académico $(93,94 \%)$, un regular rendimiento académico $(1,52 \%)$ y un bajo rendimiento académico $(4,55 \%)$.

Respecto al estado nutricional los alumnos exhibieron: bajo peso (47\%), peso normal $(37,88 \%)$, obeso $(3,03 \%)$ y con sobre peso $(12,09 \%)$.

Al relacionar el estado nutricional con el rendimiento académico por asignaturas se encontró que el 42,44\% mostró bajo peso con rendimiento académico alto; los escolares presentaron peso normal $(37,88 \%)$ y con sobre peso $(10,61 \%)$ un alto rendimiento académico mientras que el $1,52 \%$ con sobre peso mostró bajo rendimiento académico. Hay que destacar que de las seis aulas de los alumnos el 3,04\% y el 1,52\% con bajo peso exhibieron bajo rendimiento académico $(4,55 \%)$ y rendimiento académico regular $(1,52 \%)$ respectivamente.

No existe una relación significativa entre la desnutrición y el rendimiento académico de los alumnos del primer al sexto grado de primaria de la I. E. E. 18003 "Santa Rosa".

\section{RECOMENDACIONES}

Los efectos de este estudio concluyen que no existe una relación estadísticamente significativa entre las dos variables, por tal razón nos llevan a recomendar lo siguiente:

Realizar proyectos de educación sobre estilos de vida saludable, señalando la importancia de un consumo de alimentos balanceados, con un grupo multidisciplinario.

Promover programas de vida saludable para mejorar el estado nutricional del alumnado.
Proseguir la investigación, para lograr otros elementos que colaboren en una etapa nutricional adecuada e identificar los agentes que intervengan en un conveniente rendimiento académico.

Insertar a las diversas autoridades de la Regiónen en la ejecución de la investigación con la finalidad de conseguir efectos positivos.

Realizar planes estratégicos de educación sobre nutrición equilibrada e higiene de alimentos.

\section{REFERENCIAS BIBLIOGRÁFICAS}

Antúnez, C. (2010). Inteligencias múltiples: Como estudiarlas y desarrollarlas. 1ra. Edición.

Arévalo, J. Castillo, J. (2011). Relación entre el estado nutricional y el rendimiento académico en estudiantes de la institución educativa José Enrique Celis Bardales. Tarapoto, Mayo Diciembre 2011. San Martín. Perú.

Balarezo, C. (2012). Desnutrición crónica y anemia asociación con rendimiento escolar en niños y niñas de 6 a 12 años de la Escuela República de Chile. Cuenca. Ecuador.

Beltrán, R. (2015). Metodología de la Investigación Científica. Edit. CONCYTEC. Lima. Perú.

Cabezas, M., L., Salinas. (2009). Monitoreo Nacional de Indicadores Nutricionales. Dirección Ejecutiva de Vigilancia Alimentaria y Nutricional. Lima. Perú.

Campos C., Palomino H. (2007). Relación Talla, Edad y el Rendimiento Intelectual de escolares de 6 a 9 años-Acobamba. Tarma. Perú.

Canales, A. (2006). Metodología de la Investigación. 2da. Edición. Washington. EE. UU.

Chávez, M. (2008). Uso de internet y rendimiento académico de los estudiantes de la Facultad de Ciencias de la Educación y Humanidades. Universidad Nacional de la Amazonía Peruana. Iquitos. Perú.

Daza, C. (2008). Nutrición infantil y Rendimiento escolar. Colombia.

García, S. (1997). Anemia nutricional y rendimiento académico en estudiantes de Educación Primaria del C. N. Javier Pérez de Cuellar. San Juan de Lurigancho - Lima.

Guyton, A. Hall, C. (2015). Tratado de Fisiología Médica. $11^{\circ}$ Edición. España.

Haddad, J. (2004). Un análisis de desarrollo infantil en el área de Cholula con información limitada. 
Tesis Licenciatura. Economía. Departamento de Economía, Escuela de Ciencias Sociales, Universidad de las Américas Puebla. Mexico.

Hernández, R, Fernández C. Baptista L. (2015). Metodología de a Investigación. Editorial McGraw-Hill, México.

Jhonson M. (2010). Niños Mexicanos afectados por la desnutrición. México.

Largo, L. (2006). En Amazonas: Índices de desnutrición por encima del nivel nacional. Lima. Perú.

Max, L. (1999). Psicología del Aprendizaje, Noviembre 1999. Mexico.

Ministerio de Salud. (2011). Manual Simplificado. Lima. Perú.

Morales, E. Granados S. (1997). Anemia ferropénica y rendimiento escolar. Guatemala.

Mundaca, E. (2011). Factores socioeconómicos relacionados con la desnutrición aguda en niños de 3 a 5 años, Asentamiento Humano Pedro Castro alva. Chachapoyas. Perú.19 Moura, P. (2005). Estado nutricional de alumnos de 6 a 10 años de escuelas públicas $\left(1^{\circ}\right.$ e $2^{\circ}$ ciclos $)$, municipios de zona urbana de la Municipalidad de Pinhão. Escuela de Enfermería de Ribeirão Preto (EERP). Brasil.

Pajuelo J, Mirò H, Novak A. (2001). La desnutrición crónica, el sobrepeso y obesidad en niños de 6 a 9 años en áreas urbanas del Perú. Revista Diagnostico. Vol. 40.Num. 4.

Pollit, E. (2005). La desnutrición y el rendimiento escolar. Lima. Perú.

Renault, G. (2003). Factores que intervienen en el rendimiento académico de los estudiantes de psicología y psicopedagogía de la Universidad Privada del Salvador. Buenos Aires. Argentina.

Rondón, E. (2010). Comprensión lectora y rendimiento académico de los estudiantes del colegio científico Albert Einstein.

Salazar, G. (2013). Relación entre técnicas de estudio y rendimiento académico, Facultad de Enfermería-Universidad Nacional Toribio Rodriguez de Mendoza Amazonas-2011.

Salazar, J. (2014). Inteligencia emocional y rendimiento académico de los estudiantes, Facultad de Enfermería, Universidad Nacional Toribio Rodriguez de Mendoza Chachapoyas.

San Segundo, M. (2006). Datos y Cifras del Sistema Universitario. Lima. Perú.
Toro, V. (2011). El perfil del profesor universitario de Ciencias Básicas. Perú.

Tonconi, J. (2009). Factores que influyen en el rendimiento académico y la deserción de los estudiantes de la Facultad de Ingeniería Económica de la UNAPuno.

UNICEF. (2011). Datos y Cifras clave sobre Nutrición. Achievable imperative for global pregress.

Varela, C. (2006). La población uruguaya a inicios del siglo XXI. Montevideo. Uruguay.

Villa de la Portilla, A. (2003). Salud y Nutrición en los primeros años, Primera Edición, 2003.

Zarate, M. (2013). Tipo de familia y rendimiento académico de los estudiantes de la Facultad de Enfermería. Universidad nacional Toribio Rodríguez de Mendoza. Chachapoyas-2013. 